S. Kwok, M. Dopita, and R. Sutherland, eds.

\title{
Kinematics of Planetary Nebulae in M51's Tidal Tail
}

\author{
John J. Feldmeier, J. Christopher Mihos \\ Case Western Reserve University, 10900 Euclid Ave. Cleveland, OH \\ 44106, U.S.A.
}

\author{
Patrick R. Durrell, Robin Ciardullo \\ Penn State University, 525 Davey Lab, University Park, PA 16802, \\ U.S.A.
}

George H. Jacoby

WIYN Observatory, P.O. Box 26732, Tucson AZ 85726, U.S.A.

\begin{abstract}
The galaxy pair NGC 5194/95 (M51) is one of the closest and best known interacting systems. Despite its notoriety, however, many of its features are not well studied. Extending westward from NGC 5195 is a low surface brightness tidal tail, which can only be seen in deep broadband exposures. Our previous [O III] $\lambda 5007$ planetary nebulae (PN) survey of M51 recovered this tidal tail, and presented us with a opportunity to study the kinematics of a galaxy interaction in progress. We report the results of a spectroscopy survey of the PN, aimed at determining their kinematic properties. We then use these data to constrain new self-consistent numerical models of the system.
\end{abstract}

M51 is one of the most famous interacting galaxy systems, but after years of modeling (e.g., Toomre \& Toomre 1972; Hernquist 1990; Salo \& Laurikainen 2000), a full description of the encounter is still not well understood. To study this system in more detail, we completed an [O III] survey for planetary nebulae (PN) in M51 (Feldmeier, Ciardullo, \& Jacoby 1997). Surprisingly, we found a large number of PN to the west of the secondary galaxy NGC 5195 in a tidal-tail like structure (Fig. 1, left). Although this feature had been detected previously with deep photographic imaging (Burkhead 1978), it has remained unstudied until now.

To study the kinematics of the M51 system, we used the Hydra fiber spectrograph on the WIYN 3.5-m telescope to measure the radial velocities of 36 of the galaxies' halo PN. By comparing the spectra of identical PN taken in multiple setups, we find that the velocity uncertainties are less than $17 \mathrm{~km} \mathrm{~s}^{-1}$. We find a complex velocity distribution in the tidal tail, with multiple components that can be attributed to each galaxy.

To understand the kinematic properties of the PN, we constructed an Nbody model of the M51 system. We began by using earlier simulations (e.g., Hernquist 1990) which suggested that the encounter involves a mass ratio of $2: 1$, an elliptical orbit $(e \sim 0.7)$, and a relatively high $\left(i=70^{\circ}\right)$ inclination of NGC 5195's disk to the orbital plane. We then varied the orientation of the 

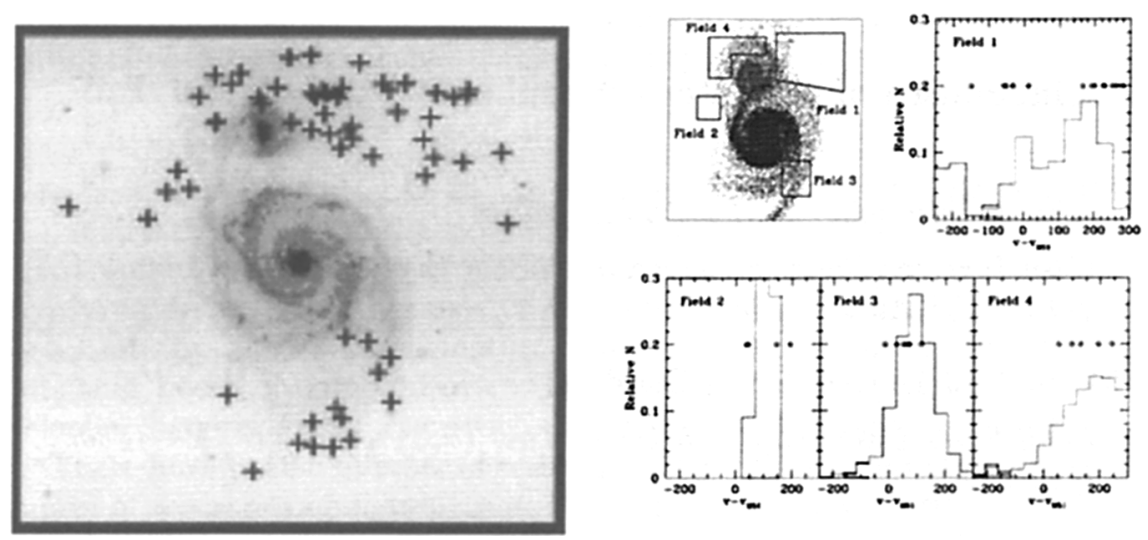

Figure 1. On the left is our [O III] $\lambda 5007$ image of the M51 system; the $64 \mathrm{PN}$ are labeled by crosses. The diagrams on the right compare the results of our $\mathrm{N}$-body models (the histograms) with the observed $\mathrm{PN}$ velocities (open pentagons).

companion to produce the optical morphology and general PN kinematics. We added a bulge to each galaxy ( $\mathrm{B}: \mathrm{D}=1 / 3)$ to suppress the bar instability in the disks. The model galaxies were constructed in the manner described by Hernquist (1993) and evolved in a self-consistent manner using TREECODE (Hernquist 1987). We found that in the best-fitting model the companion is slightly retrograde $\left(i=110^{\circ}, \omega=30^{\circ}\right)$ and the system is viewed approximately $300 \mathrm{Myr}$ after the initial encounter.

To analyze the PN velocities, we divided the galaxy model into different fields and constructed radial velocity histograms of the disk particles within each region (Fig. 1, right). The tidal tail region (Field 1) shows a broad, complex, and multi-modal structure arising from an overlapping of PN in the disk of NGC 5195 , and in the tidal tails of both M51 and NGC 5195. Fields 2-4 are quieter kinematically, and sample other tidal features in the system. With very little fine tuning, we can reconstruct the velocity distribution of the observed PN using a relatively simple interaction model.

\section{References}

Burkhead, M.S. 1978, ApJS, 38, 147

Feldmeier, J., Ciardullo, R. \& Jacoby, G. 1997, ApJ, 479, 231

Hernquist, L. 1987, ApJS, 64, 715

Hernquist, L. 1990, in Dynamics and interactions of galaxies, ed. R. Wielen (New York: Springer-Verlag), 108.

Hernquist, L. 1993, ApJS, 86, 389

Salo, H. \& Laurikainen, E. 2000, MNRAS, 319, 39

Toomre, A. \& Toomre, J. 1972, ApJ, 178, 623 